\title{
Theoretical isochrones compared to 2MASS observations: Open clusters at nearly solar metallicity
}

\author{
Ch. Bonatto ${ }^{1}$, E. Bica ${ }^{1}$, and L. Girardi ${ }^{2}$ \\ ${ }^{1}$ Universidade Federal do Rio Grande do Sul, Instituto de Física, CP 15051, Porto Alegre CEP 91501-970, RS, Brazil \\ 2 Osservatorio Astronomico di Trieste, Via Tiepolo 11, Trieste, Italia
}

Received 27 August 2003 / Accepted 14 November 2003

\begin{abstract}
We study classical disk clusters at nearly solar metallicity with ages in the range $200 \mathrm{Myr}-6 \mathrm{Gyr}$ using $2 \mathrm{MASS}$ photometry and theoretical isochrones. As a first step we employ $J, H$ and $K$ Johnson's Padova isochrones which, on a large scale, result in a good fit of the observed data. However, we find some significant deviations, especially in the red giant branch for intermediate age clusters and the low-mass main-sequence end in general. Subsequently, isochrones involving the 2MASS $J$, $H$ and $K_{\mathrm{S}}$ transmission curves were generated which account for differences of $\approx 0.02$ of the above deviations, in the sense required by the 2MASS observations. However, these corrections are not enough to fully account for the observed differences. We suggest that the remaining deviations should be accounted for by model atmosphere limitations, particularly the lack of molecular opacity data in low-temperature giants. Future models will incorporate these updated data in order to produce isochrones suitable for the whole range of stellar masses.
\end{abstract}

Key words. Galaxy: open clusters and associations: general - stars: Hertzsprung-Russell (HR) and C-M diagrams

\section{Introduction}

Understanding stellar evolution from very low to high mass stars represents one of the most important - and complex - efforts of modern Astronomy, with major consequences to vastly different research fields such as galaxy formation and cosmology. Nuclear reaction rates, radiative and conductive opacities, as well as equations of state for the whole range of physical conditions prevailing in the main classes of stars are necessary to build consistent theoretical models of stellar interiors and atmospheres. Additional simplifying approximations, such as the mixing-length theory and gray atmospheres, are also necessary and widely used in this stage. The resulting model quantities, such as luminosities, effective temperatures, and surface gravities may be converted to a given photometric system by using libraries of synthetic spectra derived from more detailed atmosphere models (e.g. Kurucz 1993; Castelli et al. 1997; see Girardi et al. 2002 for a discussion). However, crucial data on molecular opacities, particularly on the atmospheres of lowtemperature giants are scarce and/or poorly determined (see, e.g. Plez 1999; Alvarez \& Plez 1998; Alvarez et al. 2000; and Houdashelt et al. 2000a,b), which in turn may affect the conversion tables, in particular for infrared bands. Isochrones, the end-product of this task, are excellent theoretical tools to derive parameters such as age, metal content, mass function slope and distance to star systems.

Send offprint requests to: C. Bonatto, e-mail: charles@if.ufrgs.br
Recently the Two Micron All Sky Survey (hereafter 2MASS, Skrutskie et al. 1997 - available at http:// www.ipac.caltech.edu/2mass/releases/allsky/), including the Point-Source Catalogue and Atlas, has produced huge amounts of data to be explored in the coming years. The photometric system comprises Johnson's $J(1.25 \mu \mathrm{m})$ and $H(1.65 \mu \mathrm{m})$ bands with the addition of $K_{\mathrm{S}}(2.17 \mu \mathrm{m})$, slightly bluer than Johnson's $K$. The 2MASS sky coverage, homogeneity and depth will certainly make this set of filters a photometric standard reference for the future.

In the present state of the art it is fundamental to compare existing isochrones in the Johnson system with 2MASS data, followed by the generation of isochrones built with the $J, H$ and $K_{\mathrm{S}} 2 \mathrm{MASS}$ transmission curves themselves.

Among the available isochrone sets in Johnson's system are those built by the Padova group (e.g. Bertelli et al. 1994; Girardi et al. 2000), which are based on the $J, H$ and $K$ Johnson system as specified in Bessell \& Brett (1988). The Padova isochrone sets were computed with updated opacities and equations of state, and a moderate amount of convective overshoot. The basic isochrone set presented in Girardi et al. (2002) - available at http:// pleiadi.pd.astro.it/ lgirardi/isocphotsys.00/ covers a very wide range of initial masses (from 0.15 to $\sim 100 M_{\odot}$ ), metallicities, and photometric systems, being well suited for studies of clusters of all ages. As a first step in this work we test these isochrones on 2MASS observations 
Table 1. WEBDA parameters on well-studied open clusters.

\begin{tabular}{lcccccccccc}
\hline \hline Cluster & {$[\mathrm{Fe} / \mathrm{H}]$} & Age $(\mathrm{Myr})$ & $d_{\odot}(\mathrm{pc})$ & $R_{\mathrm{ex}}\left({ }^{\prime}\right)$ & $(m-M)_{V}$ & $(m-M)_{J}$ & $(m-M)_{K_{\mathrm{S}}}$ & $E(B-V)$ & $E(J-H)$ & $E\left(J-K_{\mathrm{S}}\right)$ \\
\hline Pleiades, M 45 & +0.11 & 135 & 150 & 66 & 5.98 & 5.910 & 5.895 & 0.030 & 0.009 & 0.015 \\
M 41, NGC 2287 & +0.04 & 243 & 693 & 25 & 9.29 & 9.227 & 9.214 & 0.027 & 0.008 & 0.013 \\
Praesepe, M 44, NGC 2632 & +0.14 & 729 & 187 & 72 & 6.39 & 6.412 & 6.383 & 0.009 & 0.003 & 0.004 \\
M 67, NGC 2682 & -0.15 & 2564 & 908 & 15 & 9.98 & 9.799 & 9.794 & 0.059 & 0.018 & 0.029 \\
NGC 6791 & +0.11 & 4395 & 5853 & 7 & 14.21 & 13.936 & 13.879 & 0.117 & 0.036 & 0.057 \\
\hline
\end{tabular}

Table Notes. Column 4: distance to the Sun; Col. 5: radius used for VizieR extraction.

of classical open clusters around the solar metallicity level with known parameters derived mainly from the optical domain.

In Sect. 2 we compare the Johnson isochrones with 2MASS open cluster data. In Sect. 3 we discuss discrepancies between the Johnson isochrones and the observational data. In Sect. 4 we introduce isochrones specifically calculated with the 2MASS transmission filters. In Sect. 5 we discuss remaining residuals and suggest corrections to the isochrones in terms of atmospheric opacities.

\section{2MASS open clusters and Johnson isochrones}

We selected the following well-studied open clusters for the present analysis: M41 (NGC 2287), Praesepe (M44, NGC 2632), M67 (NGC 2682), Pleiades (M 45) and NGC 6791. Parameters for these clusters, obtained from the open cluster WEBDA database (Mermilliod 1996 - http://obswww. unige.ch/webda), are compiled in Table 1 . The clusters have metallicities around the solar value and are located in regions not heavily contaminated by field stars.

We used in this study photometry from the 2MASS all-sky data release. Star extractions have been performed using the VizieR tool at http://vizier.u-strasbg.fr/ viz-bin/VizieR?-source=2MASS, using the circular mode with an extraction radius comparable to the angular dimension of each cluster, as given in Col. 5 of Table 1. The center of each extraction was as follows: M41 at J2000 $06^{\mathrm{h}} 46^{\mathrm{m}} 54^{\mathrm{s}}-20^{\circ} 44^{\prime} 00^{\prime \prime}\left(\ell=101.72^{\circ}, b=-20.73^{\circ}\right)$; Praesepe at $08^{\mathrm{h}} 40^{\mathrm{m}} 24^{\mathrm{s}}+19^{\circ} 40^{\prime} 00^{\prime \prime}\left(\ell=130.10^{\circ}, b=+19.67^{\circ}\right) ; \mathrm{M} 67$ at $08^{\mathrm{h}} 51^{\mathrm{m}} 18.0^{\mathrm{s}}+11^{\circ} 50^{\prime} 00^{\prime \prime}\left(\ell=132.82^{\circ}, b=+11.83^{\circ}\right)$; Pleiades at $03^{\mathrm{h}} 47^{\mathrm{m}} 00^{\mathrm{s}}+24^{\circ} 07^{\prime} 00^{\prime \prime}\left(\ell=56.75^{\circ}, b=+24.12^{\circ}\right)$; and NGC 6791 at $19^{\mathrm{h}} 20^{\mathrm{m}} 53^{\mathrm{s}}+37^{\circ} 46^{\prime} 30^{\prime \prime}\left(\ell=290.22^{\circ}\right.$, $b=+37.77^{\circ}$.

In the present study we will employ intrinsic diagrams (absolute magnitudes and dereddened colours according to Table 1 parameters). We present in Figs. 1 to 5 the $M_{J} \times(J-H)_{0}$ and $M_{K_{\mathrm{S}}} \times\left(J-K_{\mathrm{S}}\right)_{0} 2 \mathrm{MASS}$ colour-magnitude diagrams (CMDs) for the clusters studied in this work. The isochrone fit in each panel has been obtained by shifting the positions of the stars in each cluster according to the corresponding WEBDA parameters - observed distance modulus, reddening and absorption (Table 1). Dereddening transformations employed the ratios $\frac{E(J-H)}{E(B-V)}=0.309$ and $\frac{E\left(J-K_{\mathrm{S}}\right)}{E(B-V)}=0.488$, which were derived from absorption ratios in Schlegel et al. (1998) and the ratio $\frac{A_{K_{\mathrm{S}}}}{A_{V}}=0.118$ from Dutra et al. (2002).

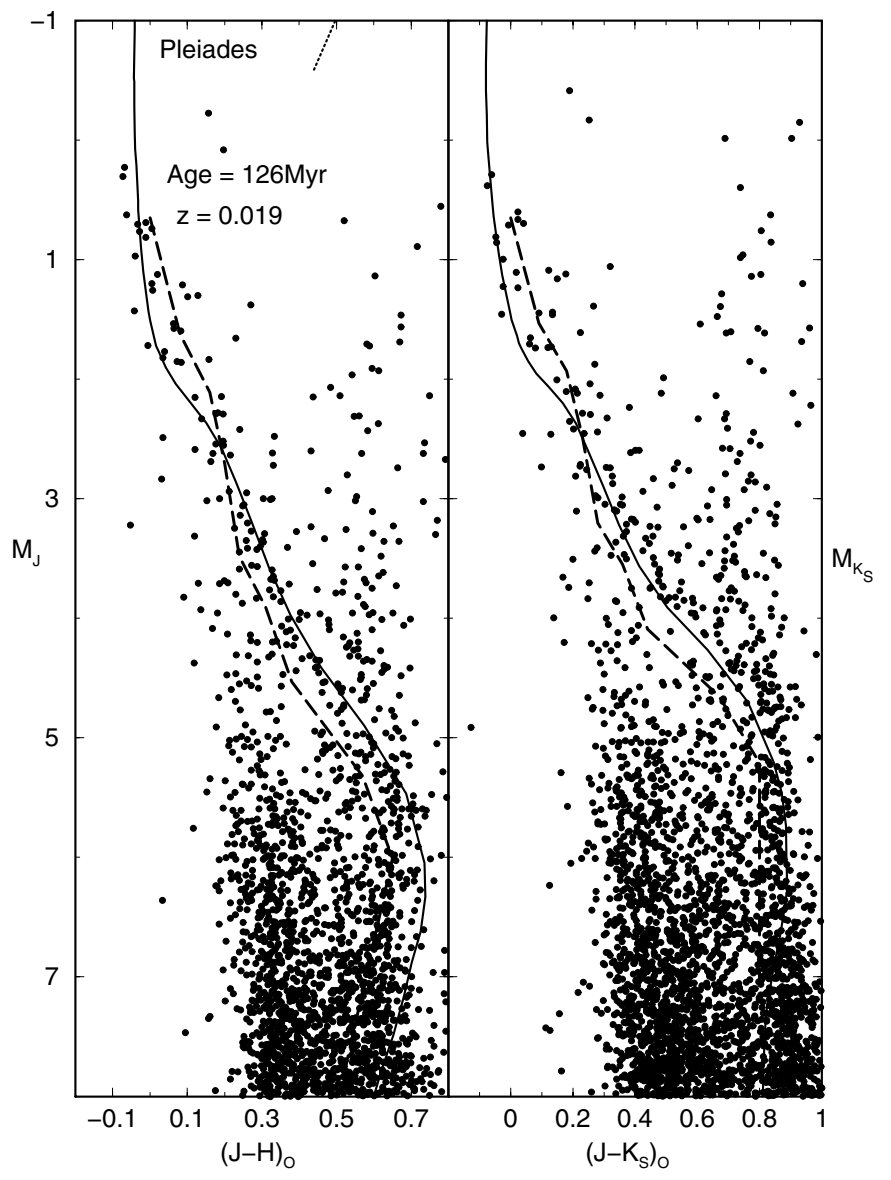

Fig. 1. 2MASS CMDs for the Pleiades (M45) built with WEBDA parameters and Padova isochrones. Left panel: $M_{J} \times(J-H)_{0}$; right panel: $M_{K_{\mathrm{S}}} \times\left(J-K_{\mathrm{S}}\right)_{0}$. The loci of solar vicinity MS (dashed-line) and Giant (dotted line) stars are also indicated as reference. Age and metallicity refer to the isochrone used. Notice that the Padova isochrone colours correspond to the Johnson photometry.

The parameters of the selected Padova Johnson's isochrones are those closest available to the WEBDA age and metallicity. We remind that the Padova isochrones are built with Johnson's $K$ band, whereas the clusters have been observed and calibrated with the 2MASS $K_{\mathrm{S}}$ filter. For comparison purposes, we also include in Figs. 1 to 5 the standard loci (Johnson photometry) of solar vicinity main-sequence (MS) and giant stars adapted from Binney \& Merrifield (1998). We recall that the Hipparcos data (Robinchon et al. 1999) give a distance to the Pleiades of about $118 \mathrm{pc}$, which corresponds to a distance modulus nearly 0.3 mags smaller than that used 


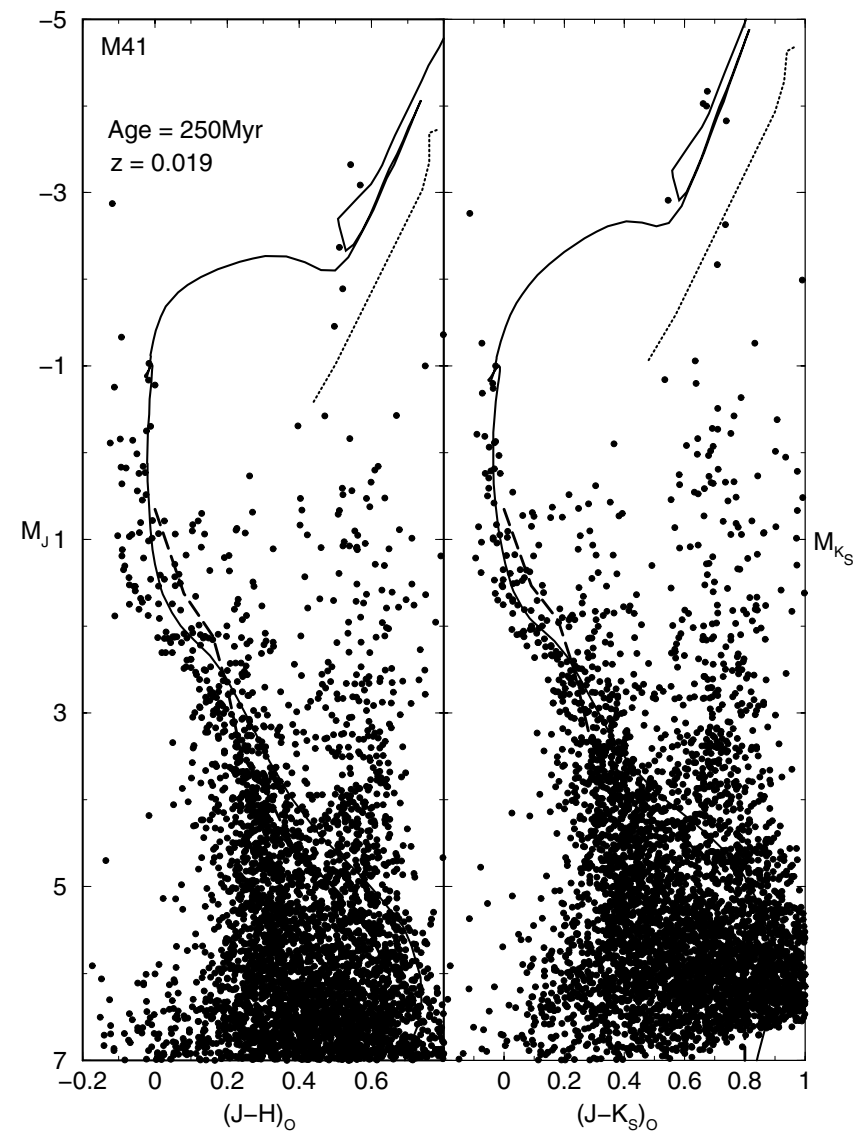

Fig. 2. Same as Fig. 1 for M 41 (NGC 2287).

in the present isochrone superposition to the 2MASS data (Fig. 1). Applying the Hipparcos modulus would degrade the solution, particularly for the $M_{J} \times(J-H)_{0}$.

\section{Comparison of Johnson isochrones and observations}

Despite field contamination, the low MS can be studied in the Pleiades (Fig. 1) and Praesepe (Fig. 3), since they are nearby clusters. Massive giants can be studied in the $250 \mathrm{Myr}$ M 41 (Fig. 2); the giant branch and clump can be assessed for the intermediate-age clusters M 67 (Fig. 4) and NGC 6791 (Fig. 5). The MS can be studied in all clusters except NGC 6791, which is too far from the Sun. Inferences on the turnoff can be made for M 41, Praesepe and M 67.

We point out that the distance modulus for NGC 6791 given by WEBDA was too small by an amount of 0.8 to fit the data. Consequently, we needed to apply a correction of $\Delta(m-M)=$ +0.8 to stars for both magnitude axes in order to reconcile observation and isochrone. The WEBDA parameters for M 67 give a non-satisfactory fit of the turnoff region (Fig. 4). In this age range, the turnoff shape strongly depends on age. Indeed, with isochrones in the range 2.8-3.2 Gyr, somewhat older than WEBDA's $2.5 \mathrm{Gyr}$, a better solution to the turnoff is obtained. We show this new fit in Fig. 6, which will be that subsequently used in the analyses. A more detailed analysis of M67 using 2MASS photometry is presented in Bonatto \& Bica (2003), in

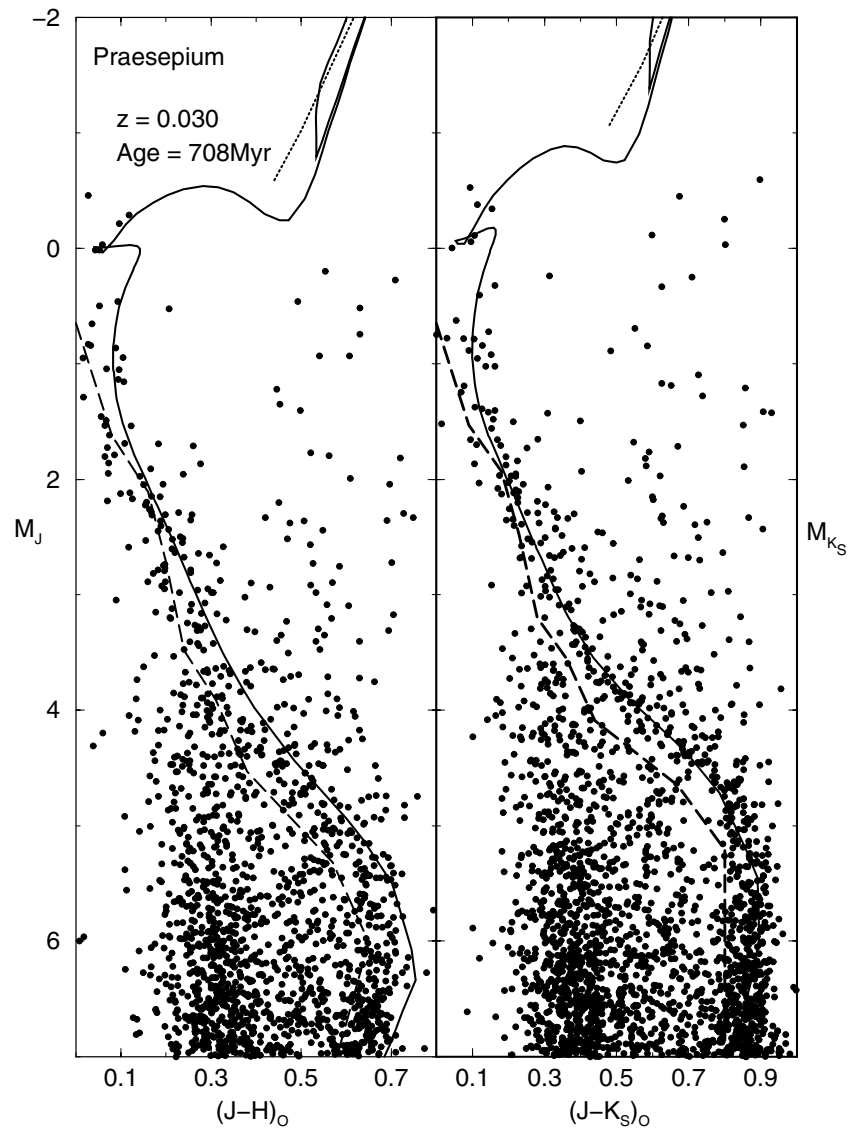

Fig. 3. Same as Fig. 1 for Praesepe (NGC 2632).

which we derive an age of $3.2 \pm 0.4 \mathrm{Gyr}$, null reddening and a distance to the Sun of $d_{\odot} \approx 0.87 \pm 0.04 \mathrm{kpc}$.

\subsection{The main sequence}

A qualitative analysis of Figs. 1 to 6 shows that the overall isochrone fits are to first order representative of the observations. Particularly worthy of notice is that the isochrones give a good general description of the main sequence of the Pleiades (Fig. 1) and Praesepe (Fig. 3), over a large range of absolute magnitudes (from $M_{K_{\mathrm{S}}} \sim 1$ to $M_{K_{\mathrm{S}}} \sim 6$ ). They clearly predict the most prominent changes in the main sequence slope, occurring at about $M_{J} \simeq 4, M_{K_{\mathrm{S}}} \simeq 3.5$, and $M_{J} \simeq 5.5, M_{K_{\mathrm{S}}} \sim 5$. This description of the main sequence by the models is better than the empirical curve given by Binney \& Merrifield (1998).

However, the isochrones do not reproduce some details of the observed CMDs, particularly with respect to the lower MS and, to a lesser extent, the giant branch. This effect does not systematically depend on age and metallicity. For example, the isochrone fit with the WEBDA parameters for the Pleiades, the youngest cluster of this sample with age $\approx 135 \mathrm{Myr}$ (Fig. 1), is considerably redder than the observed lower-MS stars in the $(J-H)_{0}$ colour (by $\approx 0.10 \mathrm{mag}$ ), at magnitudes $M_{J} \gtrsim 5.5$. On the other hand, this part of the main sequence is well described by the solar vicinity stars (the Binney \& Merrifield 1998 relation in the left panel of Fig. 1). In $\left(J-K_{\mathrm{S}}\right)_{0}$ (right panel), both sequences are compatible with the observed stars. For Praesepe 


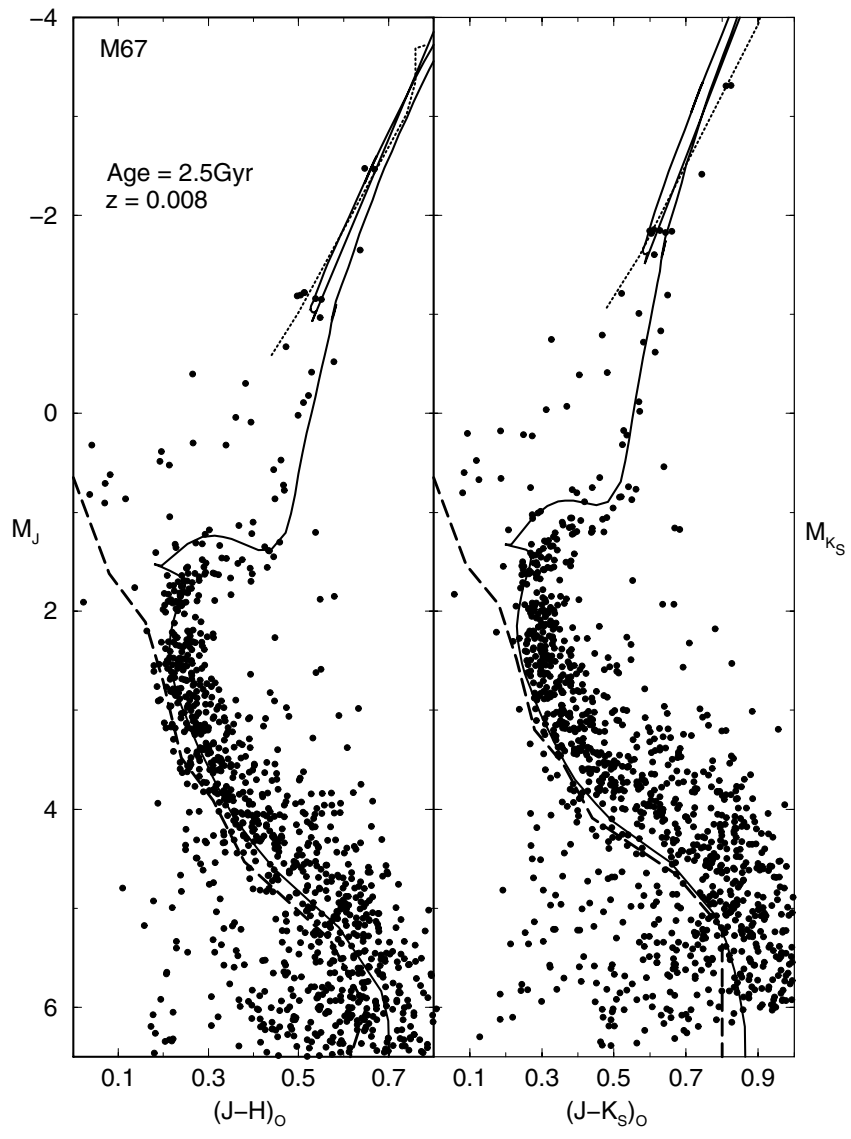

Fig. 4. Same as Fig. 1 for M 67 (NGC 2682).

(Fig. 3), the Padova isochrone is also redder in $(J-H)_{0}$ (by $\approx 0.07 \mathrm{mag}$ ) than the observed lower MS, while the agreement is good in $\left(J-K_{\mathrm{S}}\right)_{0}$.

\subsection{Giants and sub-giants}

Although there occur only a few massive giants in the CMDs of M 41 (Fig. 2), they appear to be well described by the Padova isochrones both in $(J-H)_{0}$ and $\left(J-K_{\mathrm{S}}\right)_{0}$.

Intermediate-age giants are well adjusted in both colours for M 67 (Fig. 4) and for NGC 6791 (Fig. 5).

The isochrones for M67 and NGC6791 are too red in $(J-H)_{0}$ by $\approx 0.07$ with respect to the sub-giant branches (Figs. 6 and 5, respectively).

The giant clump is well described for M67 (Fig. 6). For NGC 6791, the $\left(J-K_{\mathrm{S}}\right)_{0}$ fit is good, but the corresponding isochrone locus is too red by $\approx 0.07$ in $(J-H)_{0}$.

\subsection{The turnoff}

Considering uncertainties in the parameters, the turnoff region is well described for the Praesepe and M67. For M41, the $\left(J-K_{\mathrm{S}}\right)_{0}$ solution is suitable, while there are deviations in the $(J-H)_{0}$ solution (Fig. 2).

For clusters younger than $\approx 1 \mathrm{Gyr}$, the MS fits are suitable except for the $\left(J-K_{\mathrm{S}}\right)_{0}$ solution for the Pleiades, in which the isochrone is too faint by about $0.25 \mathrm{mag}$. The solar vicinity

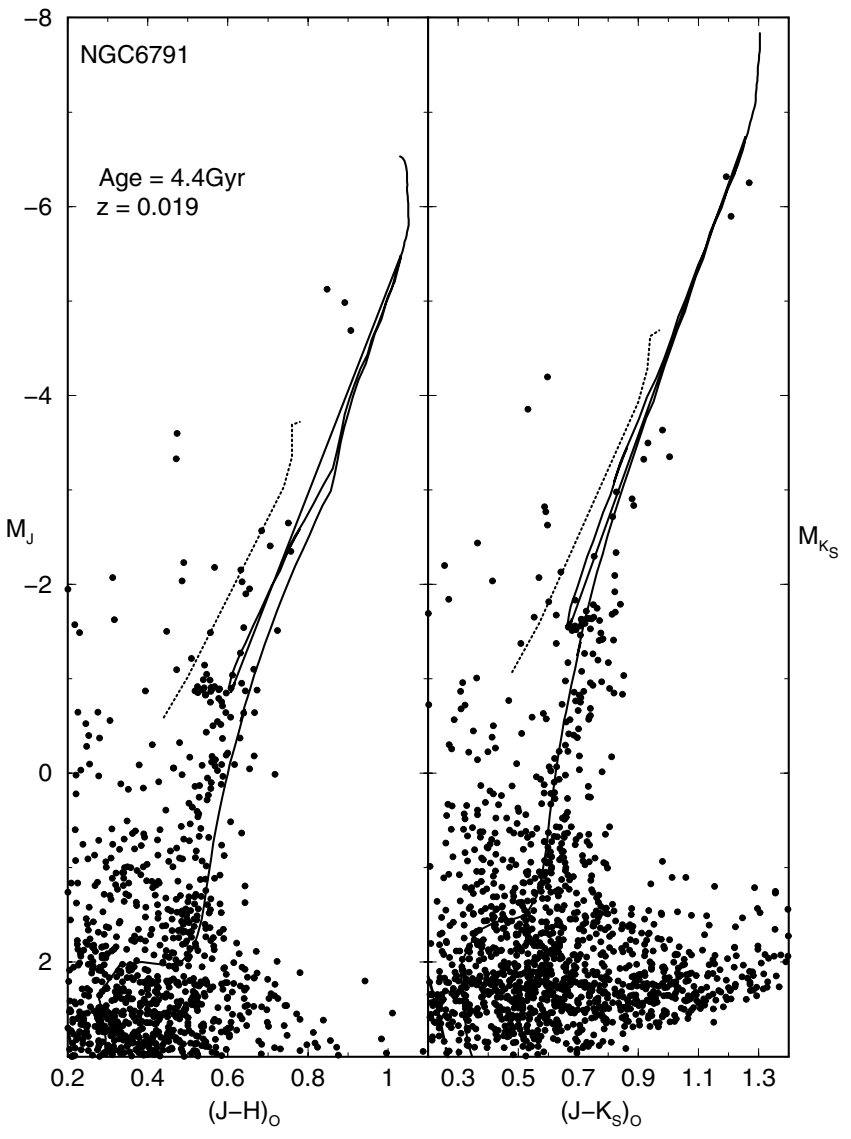

Fig. 5. Same as Fig. 1 for NGC 6791. With respect to the WEBDA distance modulus, we needed to apply an additional correction of $\Delta(m-M)=+0.8$ to the stars for both magnitude axes in order to reconcile observation and isochrone.

sequence also describes well the observed MSs, except those of the Praesepe (Fig. 3) where it is too faint by about 0.25 mag. In $M_{K_{\mathrm{S}}} \times\left(J-K_{\mathrm{S}}\right)_{0}$, the solar vicinity MS is also too faint (Fig. 1).

At intermediate ages (M67), the isochrones turned out bluer than the observed stars, by about 0.04 in $(J-H)_{0}$ and 0.08 in $\left(J-K_{\mathrm{S}}\right)_{0}$. In this case we suspect that most of the discrepancy is not due to the models, since M 67 is known to have at least a fraction of $38 \%$ of binaries (Montgomery et al. 1993). However, the behaviour is not exactly the same in the $(\mathrm{J}-H)_{0}$ and $\left(J-K_{\mathrm{S}}\right)_{0}$ diagrams (Fig. 6), which might be accounted for by the models.

The above series of differences between theory and observation cannot be fully ascribed to simple observational in the uncertainties parameter, instead they appear in most cases to reflect some systematic effects in the theory which should be properly taken into account.

\section{Padova isochrones with 2MASS transmission filters}

It is fundamental for 2MASS data analysis consistency that a new set of isochrones be built taking into account specifically the transmission filters employed by $2 \mathrm{MASS}$. 


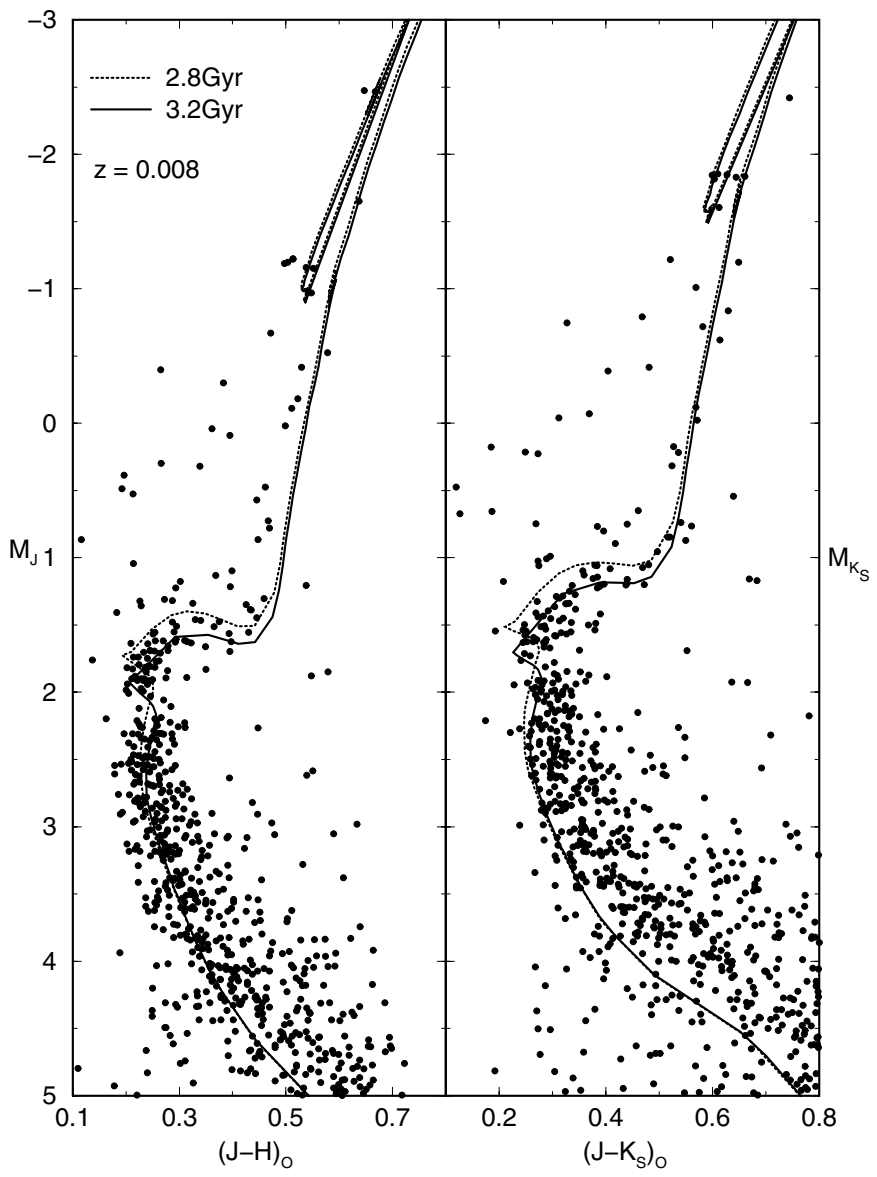

Fig. 6. A best-fit for M 67 is obtained with somewhat older isochrones (with respect to WEBDA's data) which, however, do not fix the MS and giant branch deviations. Left panel: $M_{J} \times(J-H)_{0}$; right panel: $M_{K_{\mathrm{S}}} \times\left(J-K_{\mathrm{S}}\right)_{0}$.

The 2MASS transmission curves have been downloaded from the site http://www.ipac.caltech.edu/ 2mass/releases/second/doc/ sec3_1b1.html). Figure 7 compares them with Johnson filters (cf. Bessell \& Brett 1988), and a few representative stellar spectra. A new set of 2MASS $J H K_{\mathrm{s}}$ isochrones was generated in the same way as in Girardi et al. $(2002)^{1}$. In Fig. 8 we compare the present new $J, H$ and $K_{\mathrm{S}}$ set with that of Johnson's (Girardi et al. 2002) in a $M_{K_{\mathrm{S}}} \times\left(J-K_{\mathrm{S}}\right) \mathrm{CMD}$ for solar metallicity isochrones with ages $63 \mathrm{Myr}, 250 \mathrm{Myr}, 2 \mathrm{Gyr}$ and $10 \mathrm{Gyr}$.

The 2MASS transmission filters produced isochrones very similar to the Johnson ones, with small $\left(K-K_{\mathrm{S}}\right)$ differences which are slightly dependent on age. For intermediate-to-old ages, $\left(K-K_{\mathrm{S}}\right)>0$ for the lower+upper main sequences and turnoff, while for young ages $\left(K-K_{\mathrm{S}}\right)>0$ for the lower main sequence and $\left(K-K_{\mathrm{S}}\right)<0$ for the upper main sequence and turnoff. $\left(K-K_{\mathrm{S}}\right)<0$ in the giant branch for all ages.

In the insets of Fig. 8 we show blowups of the turnoff and giant branch regions, where $K$ and $K_{\mathrm{S}}$ differ significantly. In the turnoff region of the $10 \mathrm{Gyr}$ isochrones the difference is

1 The new 2MASS $J H K_{\mathrm{s}}$ isochrones are made available at http:// pleiadi.pd.astro.it/ lgirardi/isoc_photsys.00/, together with the previous Padova database.

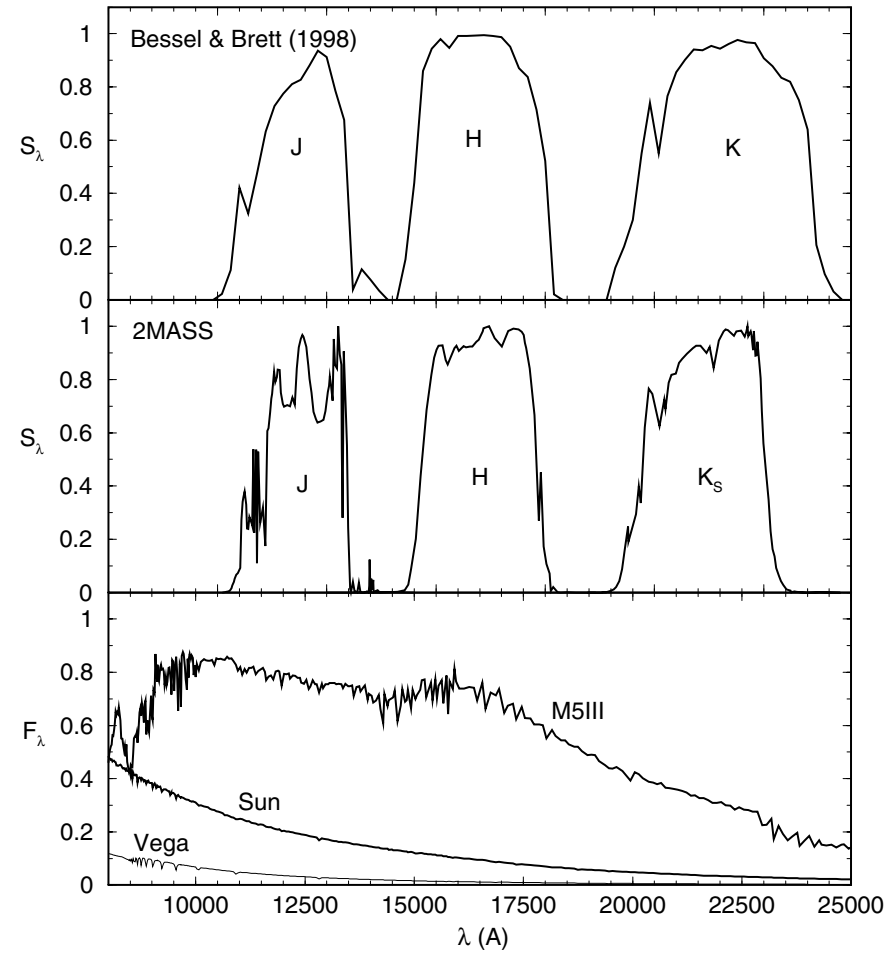

Fig. 7. 2MASS $J, H$ and $K_{\mathrm{S}}$ transmission curves (middle panel) are compared to those of the Johnson system (top panel); spectra of representative stars are shown in the bottom panel.

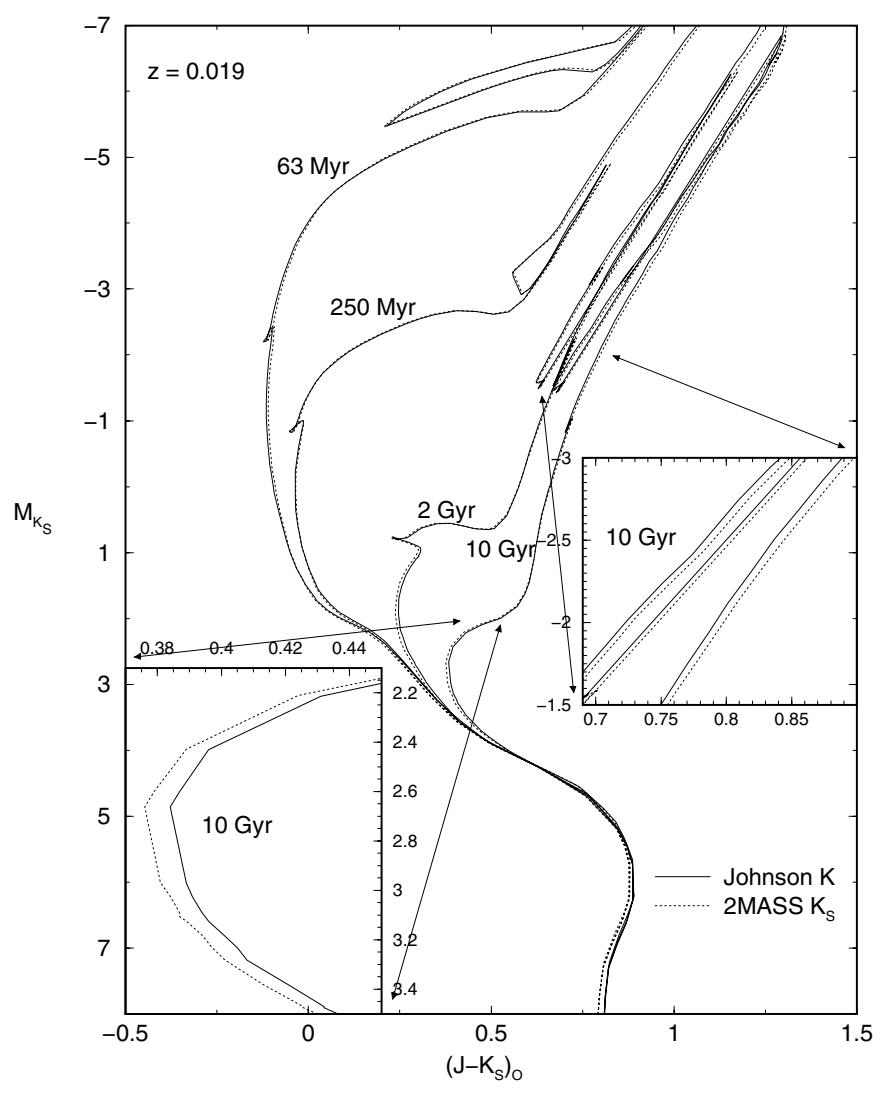

Fig. 8. Comparison of Johnson $K$ with 2 MASS $K_{\mathrm{S}}$ isochrones for different ages and solar metallicity. Insets show blowups of the turnoff and giant branch regions of the $10 \mathrm{Gyr}$ isochrone. Differences $\left(K-K_{\mathrm{S}}\right)$ are $\approx+0.02$ at the turnoff and $\approx-0.01$ at the giant branch. 


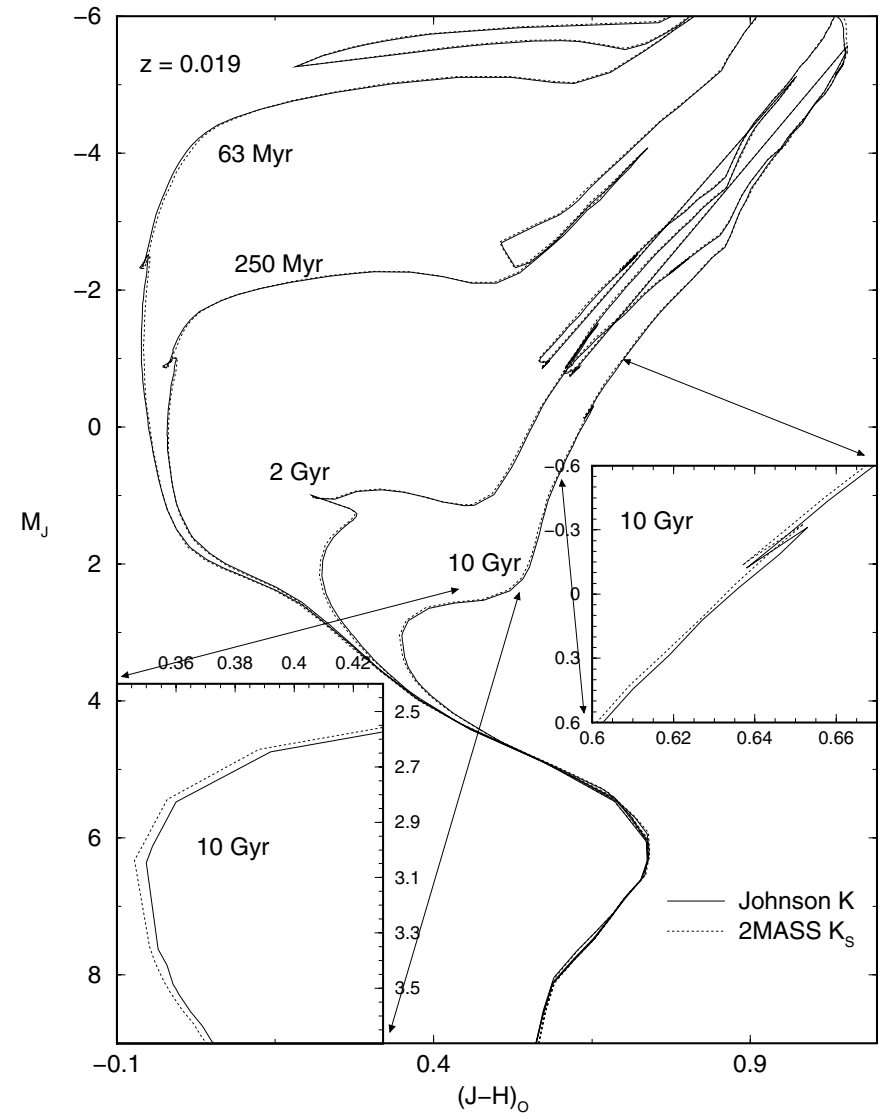

Fig. 9. Same as Fig. 8 for the $J$ and $H$ filters. Differences in $(J-H)_{0}$ colour are at most 0.01 throughout.

$\left(K-K_{\mathrm{S}}\right) \approx+0.02$ while at the giant branch $\left(K-K_{\mathrm{S}}\right) \approx-0.01$. For the low-mass end, the differences found are $\left(K-K_{\mathrm{S}}\right) \approx$ +0.02 for all ages.

With respect to the $J$ and $H$ filters, the present 2MASS set produced isochrones which differ at most 0.01 in $(J-H)_{0}$ (Fig. 9).

A comparison of the 2MASS observations (Sect. 2) and the new $J, H$ and $K_{\mathrm{S}}$ isochrones shows that the corrections of $\approx+0.02$ are in general in the required sense, however, significant deviations still remain which probably arise in the theory.

\section{Concluding remarks}

In this work we have compared $J, H$ and $K_{\mathrm{S}}$ 2MASS observations of reference disk open clusters at nearly solar metallicity, with previously available $J, H$ and $K$ Padova isochrones. On a large scale, the Johnson isochrones produce a reasonable representation of the main features observed on the CMDs of the clusters. However, some significant differences between the isochrones and observations arise which cannot be ascribed to observational parameters such as reddening, distance, age and metallicity uncertainties.

As a second step, we generated new isochrones specifically built with the 2MASS filter transmission curves. Empirical corrections were applied to the original models, thus producing a new $J, H$ and $K_{\mathrm{S}}$ set of isochrones which, in principle, should be more appropriate to study open clusters of nearly solar metallicity observed with 2MASS. Although some small improvements with respect to the Johnson's $J H K$ isochrones have been obtained, this new set of isochrones (presented in Sect. 4) does not appreciably change the description of the 2MASS observations. In short, most of the discrepancies pointed out in Sect. 3 still remain, and new ones appeared, which could be related to data deficiency on atmospheric opacities in the models.

Further improvements in the $J, H$ and $K_{\mathrm{S}}$ isochrones with respect to the observations certainly involve a few small changes on the transformation tables from stellar model parameters to observational quantities, since the lack of molecular opacity data in Kurucz's (1993) models may account for some (or even most) of the remaining deviations.

Acknowledgements. This publication makes use of data products from the Two Micron All Sky Survey, which is a joint project of the University of Massachusetts and the Infrared Processing and Analysis Center/California Institute of Technology, funded by the National Aeronautics and Space Administration and the National Science Foundation. We employed data from CDS/Simbad/VizieR (Strasbourg) and also made use of the WEBDA open cluster database. We acknowledge support from the Brazilian Institution CNPq. We thank Dr. D. Soderblom for useful comments.

\section{References}

Alvarez, R., Lançon, A., Plez, B., \& Wood, P. R. 2000, A\&A, 353, 322

Alvarez, R., \& Plez, B. 1998, A\&A, 330, 1109

Bertelli, G., Bressan, A., Chiosi, C., Fagotto, F., \& Nasi, E. 1994, A\&AS, 106, 275

Bessell, M. S., \& Brett, J. M. 1988, PASP, 100, 1134

Binney, J., \& Merrifield, M. 1998, in Galactic Astronomy (Princeton, NJ: Princeton University Press) (Princeton series in astrophysics) QB857.B522

Bonatto, C. J., \& Bica, E. 2003, A\&A, 405, 525

Castelli, F., Gratton, R. G., \& Kurucz, R. L. 1997, A\&A, 318, 841

Dutra, C. M., Santiago, B. X., \& Bica, E. 2002, A\&A, 381, 219

Girardi, L., Bertelli, G., Bressan, A., et al. 2002, A\&A, 391, 195

Girardi, L., Bressan, A., Bertelli, G., \& Chiosi, C. 2000, A\&AS, 141, 371

Houdashelt, M. L., Bell, R. A., Sweigart, A. V., \& Wing, R. F. 2000a, AJ, 119, 1424

Houdashelt, M. L., Bell, R. A., \& Sweigart, A. V. 2000b, AJ, 119, 1448

Kurucz, R. L. 1993, in Model Atmospheres, VizieR On-line Data Catalog: VI/39

Mermilliod, J. C. 1996, in The origins, evolution, and destinies of binary stars in clusters, ASP Conf. Ser., 90, 475

Montgomery, K. A., Marschall, L. A., \& Janes, K. A. 1993, AJ, 106, 181

Plez, B. 1999, in Asymptotic Giant Branch Stars, ed. T. Le Bertre, A. Lebre, \& C. Waelkens, IAU Symp., 191, 75

Robinchon, N., Arenou, F., Mermilliod, J.-C., \& Turon, C. 1999, A\&A, 345, 471

Schlegel, D. J., Finkbeiner, D. P., \& Davis, M. 1998, ApJ, 500, 525

Skrutskie, M., Schneider, S. E., Stiening, R., et al. 1997, in The Impact of Large Scale Near-IR Sky Surveys, ed. F. Garzon et al. (Netherlands: Kluwer), 210, 187 